\title{
AN IMPLEMENTATION OF RECOVERY ALGORITHM FOR FAULT NODES IN A WIRELESS SENSOR NETWORK
}

\author{
Anuradha M S ${ }^{1}$,DeepaPatil ${ }^{2}$ \\ ${ }^{1}$ Associate Prof, Deptof ECE, Guru Nanak Dev Engg. College, Bidar, Karnataka, India \\ ${ }^{2}$ Dept of ECE, Guru Nanak Dev Engineering College, Bidar, Karnataka, India
}

\begin{abstract}
This paper proposes a fault node recovery algorithm to enhance the lifetime of a wireless sensor network when some of the sen sor nodes shut down. The algorithm is based on the fault node route discovery and fault node detection algorithm. The algorithm can result in fewer replacements of sensor nodes and more reused routing paths. In our simulation, the proposed algorithm increases the number of active nodes, reduces the rate of power consumption, and reduces the rate of energy consumption, number of hops and time taken.
\end{abstract}

Keywords: Grade diffusion algorithm, Wireless Sensor Networks (WSN), Fault node route discovery and Fault node detection.

\section{INTRODUCTION}

Wireless technologies have revolutionized the world of communications. It started with the use of radio receivers or transceivers for use in wireless telegraphy early on and now the term wireless is used to describe technologies such as the cellular networks and wireless broadband Internet. Although the wireless medium has limited spectrum along with a few other constraints as compared to the guided media, it provides the only means of mobile communication. Wireless Sensor Network (WSN) is type of network which consists of collection of tiny device called sensors nodes. In real wireless sensor networks, the sensor nodes use battery power supplies and thus have limited energy resources. Recent advances in micro processing, wireless andbattery technology, and smart sensors have enhanceddata processing [3], wireless communication, anddetection capability. In sensor networks, each sensor node has limited wireless computational power to process and transfer the live data to the base station or data collection center[2], [5]. Therefore, to increase the sensor area and the transmission area [1] the wireless sensor network usually contains many sensor nodes. Generally, each sensor node has a low level of battery power that cannot be replenished. When the energy of a sensor node is exhausted, wireless sensor network leaks will appear, and the failed nodes will not relay data to the other nodes during transmission processing.Thus, the other sensor nodes will be burdened with increased transmission processing. The use of WSN networks also introduces additional security challenges that have to be dealt with.

In the WSN, reduction of energy consumption is very important for each sensor node because it can extend WSN lifetime. The Wireless sensor network is a collection of sensors that are spread over large geographic area. Since sensors are widely spread and large in number, the occurrences of faults in the network are also more. Hence to detect the fault node and to replace the fault node an efficient algorithm is proposed.

This paper proposes a fault node recovery (FNR) algorithm to enhance the lifetime of a wireless sensor network when some of the sensor nodes shut down, either because they no longer have battery energy or they have reached their operational threshold. Using the FNR algorithm can result in fewer replacements of sensor nodes and more reused routing paths. Thus, the algorithm not only enhances the WSN lifetime but also reduces the cost of replacing the sensor nodes[5].

\section{RELATED WORK}

The traditional approaches to sensor network routing include the directed diffusion (DD) [1] algorithm and the grade diffusion (GD) [7] algorithm. The algorithm proposed in this paper is based on the GD algorithm, with the goal of replacing fewer sensor nodes that are inoperative or have depleted batteries, and of reusing the maximum number of routing paths. These optimizations will ultimately enhance the WSN lifetime and reduce sensor node replacement cost.

\subsection{Directed Diffusion Algorithm}

A series of routing algorithms [1] for wireless sensor networks, the Directed Diffusion (DD) algorithm have been proposed in recent years. The goal of the DD algorithm is to reduce the data relay transmission counts for power 
management. The DD algorithm is a query-driven transmission protocol. The collected data is transmitted only if it matches the query from the sink node. In the DD algorithm, the sink node provides the queries in the form of attributevalue pairs to the other sensor nodes by broadcasting the query packets to the whole network. Subsequently, the sensor nodes send the data back to the sink node only when it fits the queries.

\subsection{Grade Diffusion Algorithm}

The Grade Diffusion (GD) algorithm [7] is used to improve the ladder diffusion algorithm using ant colony optimization (LD-ACO) for wireless sensor networks [6]. The GD algorithm not only creates the routing for each sensor node but also identifies a set of neighbour nodes to reduce the transmission loading. Each sensor node can select a sensor node from the set of neighbor nodes when its grade table lacks a node able to perform the relay. The GD algorithm can also record some information regardingthe data relay. Then, a sensor node can select a node with a lighter loading or more available energy than the other nodes to perform the extra relay operation. That is, the GD algorithm updates the routing path in real time, and the event data is thus sent to the sink node quickly and correctly.

The Grade Diffusion algorithm overcomes the disadvantages of Direct Diffusion algorithm by broadcasting the neighbors to only first neighbor set. After that nodes are picked up based on hop count or rules and the amount of RREQ exchange is reduced hence amount of power required is less as compared to Direct Diffusion. However problem still persist as the number of routes discovered increases the battery power decreases and node becomes obsolete sooner.

Whether the DD or the GD algorithm is applied, the grade creatingpackages or interested query packets must first be broadcast. Then, the sensor nodes transfer the event data to the sink node, according to the algorithm, when suitable events occur. The sensor routing paths are shown in Fig. 1.

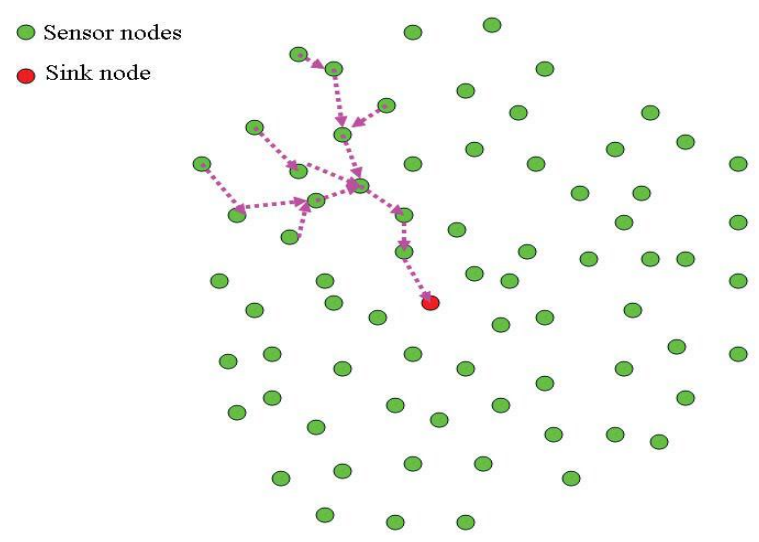

Fig. 1 Wireless sensor node routing

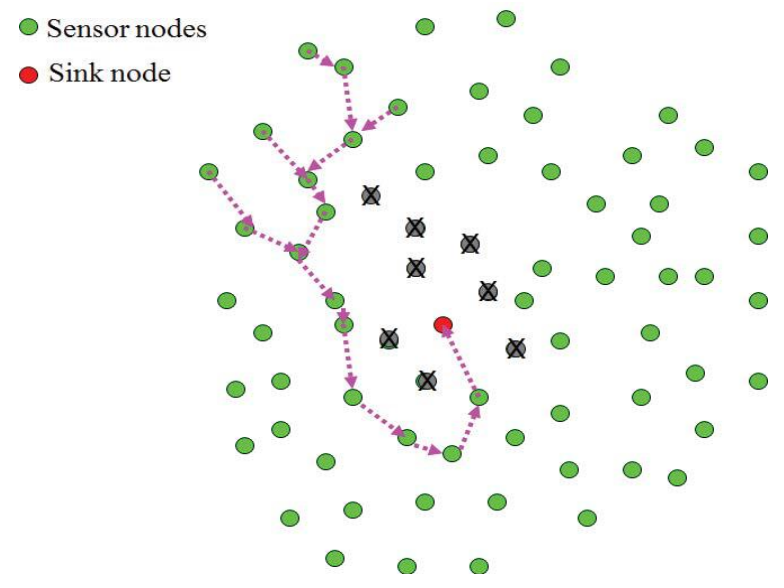

Fig 2 Wireless sensor node routing path when some nodes are not functioning

The WSN may fail due to a variety of causes, including the following: the routing path might experience a break; the WSN sensing area might experience a leak; the batteries of some sensor nodes might be depleted, requiring more relay nodes; or the nodes wear out after the WSN has been in use a long period of time. In Fig. 2, the situation in which the outside nodes transfer event data to the sink node viathe inside nodes (the sensor nodes near the sink node) in a WSN illustrate the accommodation measures for non-working nodes. The inside nodes thus have the largest data transmission loading, consuming energy at a faster rate. If all the inside nodes deplete their energy or otherwise cease to function, the event data can no longer be sent to the sink node, and the WSN will no longer function. The power consumption of the sensor nodes in WSNs is unavoidable. This paper, however, proposes an algorithm to search for and replace fewer sensor nodes and to reuse the most routing paths[5].

\section{CURRENT APPROACH}

In the current approach, a route discovery approach is proposed which reduces amount of power consumption and number of nodes becoming obsolete (dead) will be less as compared to Grade Diffusion algorithm. The proposed algorithm will also determine set of nodes known as "grades" which has two values namely 0 or 1 . Each node will become 0 if battery is greater than threshold otherwise it will be 1 . This process of finding the set of nodes whose battery power is less than threshold is called Fault Node Determination. The nodes will be replaced with new nodes of same node id this process is called Fault Node Recovery. 


\subsection{System Architecture}

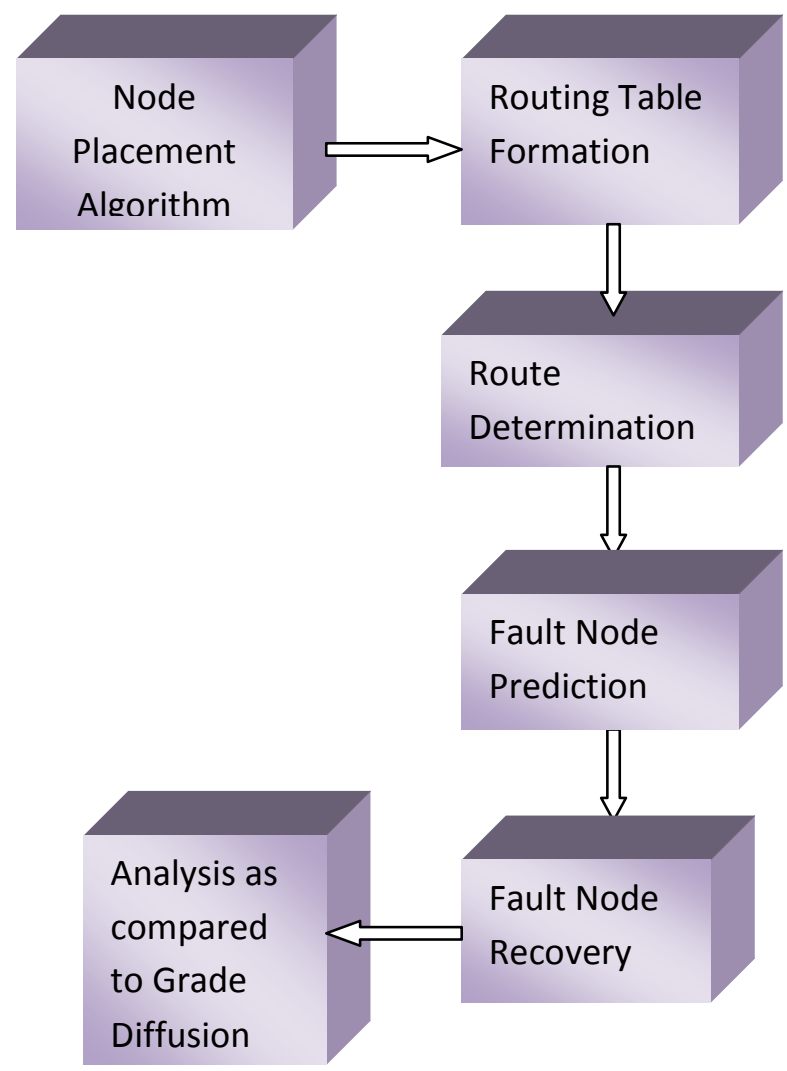

Fig 3 Block diagram

The system architecture of the implementation is as shown in the Fig.3. The nodes are deployed bounded within the area limits.

1. Node Deployment Algorithm: This algorithm is responsible for deployment of nodes in a particular area. This will position the nodes in the given area.

2. Routing Table Formation: This is the algorithm which is used to form routing tables for each of the nodes. The routing table will contain information about other nodes in the network in terms of node id and distance of each node with respect to other nodes in the network.

3. Route Determination: This is the process which involves determining the route from the source node to destination node with the aid of using the control packets and the route must be found in such a way that battery consumption is reduced and overall network lifetime is also increased.

4.Node Failure Detection: This is the process in which the node's whose battery power is below than certain threshold is determined.

5.Node Recovery: This is the process by which the nodes whose battery power is below threshold are determined and replaced with the new nodes but with same node id.

\section{SIMULATION}

In this project, the grade diffusion algorithm and fault node recovery algorithm are implemented. The various parameters such as energy consumption in $\mathrm{mJ}$, power consumption in $\mathrm{mW}$, number of hops, number of active nodes, number of dead nodes, time taken, node life time are calculated and comparsion of grade diffusion algorithm with the fault node recovery algorithm with above parameters. Hence from the output result of comparsion, the performance of fault node recovery algorithm is much better than the grade diffusion algorithm

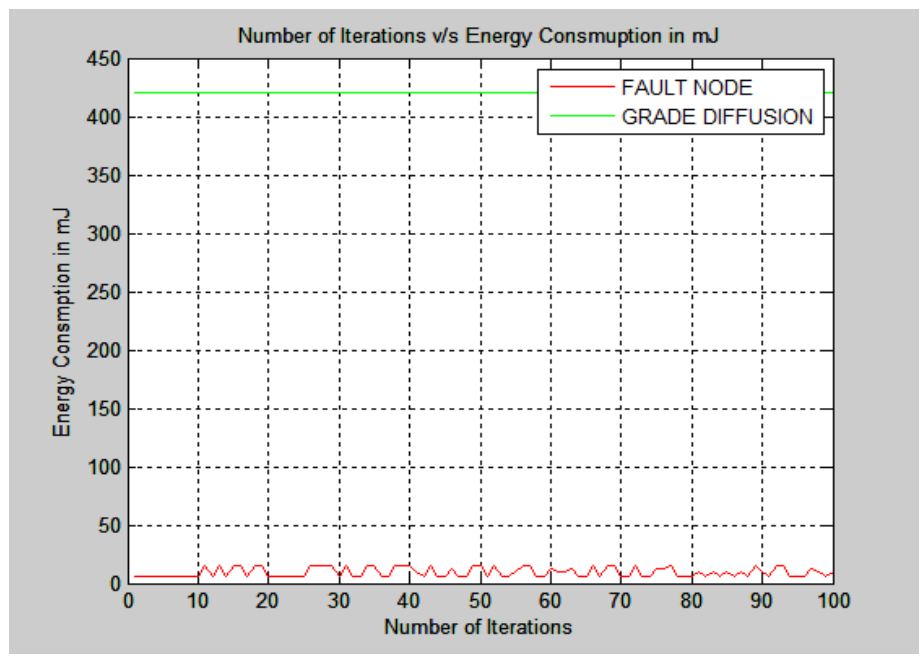

Fig.4.comparsion of the Energy consumption of fault node recovery algorithm with grade diffusion algorithm

Fig.4 shows the Energy Consumption for the routes discovered using Grade Diffusion and Fault Node and from the results one can prove Fault Node has less Energy Consumption as compared of Grade Diffusion

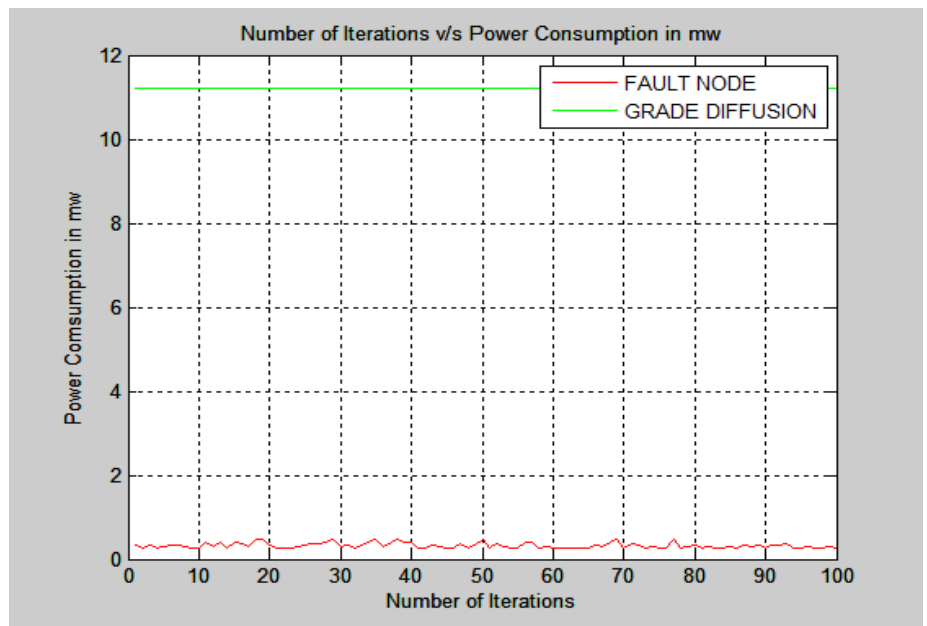

Fig.5.comparsion of power consumption of fault node recovery algorithm with grade diffusion algorithm 
Fig.5 shows the Power Consumption for the routes discovered using Grade Diffusion and Fault Node and from the results one can prove Fault Node has less Power Consumption as compared of Grade Diffusion.

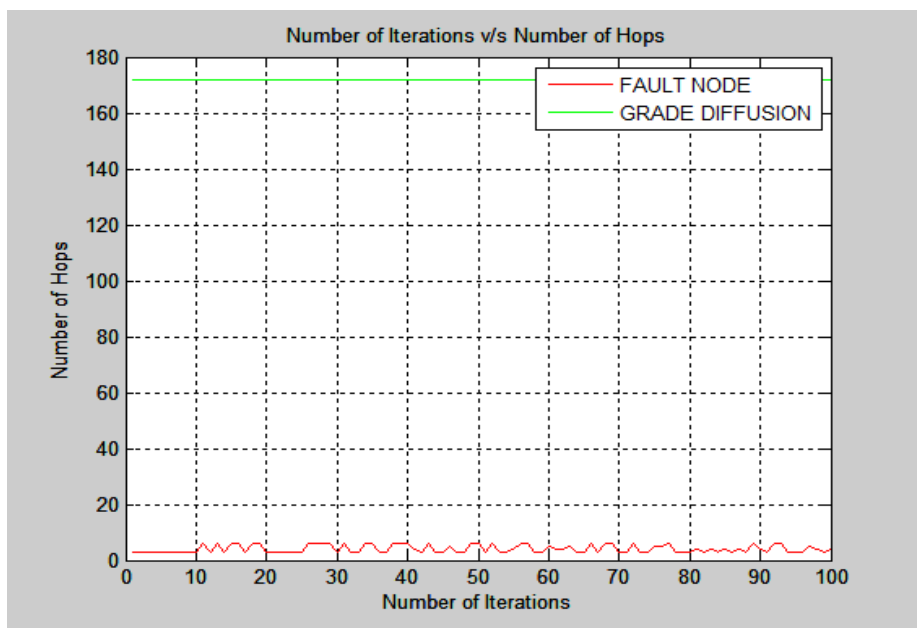

Fig.6.comparsion of the Number of hops of fault node recovery algorithm with grade diffusion algorithm

Fig.6 shows the Number of Hops for the routes discovered using Grade Diffusion and Fault Node and from the results one can prove Fault Node has less number of hops as compared of Grade Diffusion

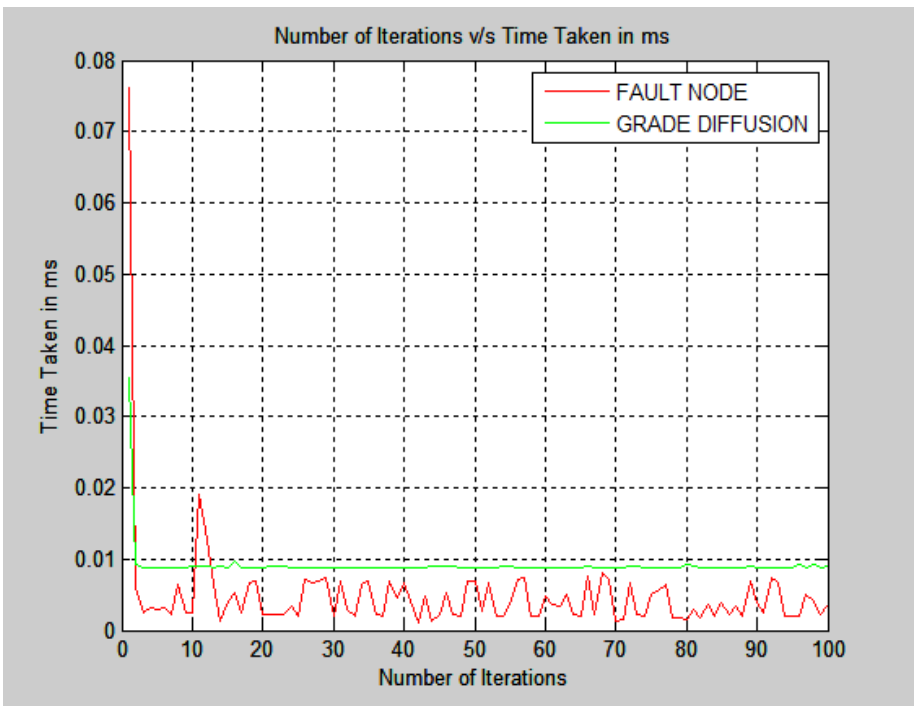

Fig.7.comparsion of time taken in ms for fault node recovery algorithm with grade diffusion algorithm

Fig.7 shows the time taken for routes discovered for the grade diffusion and fault node and from the results fault node recovery algorithm takes less time as compared with the grade diffusion algorithm.

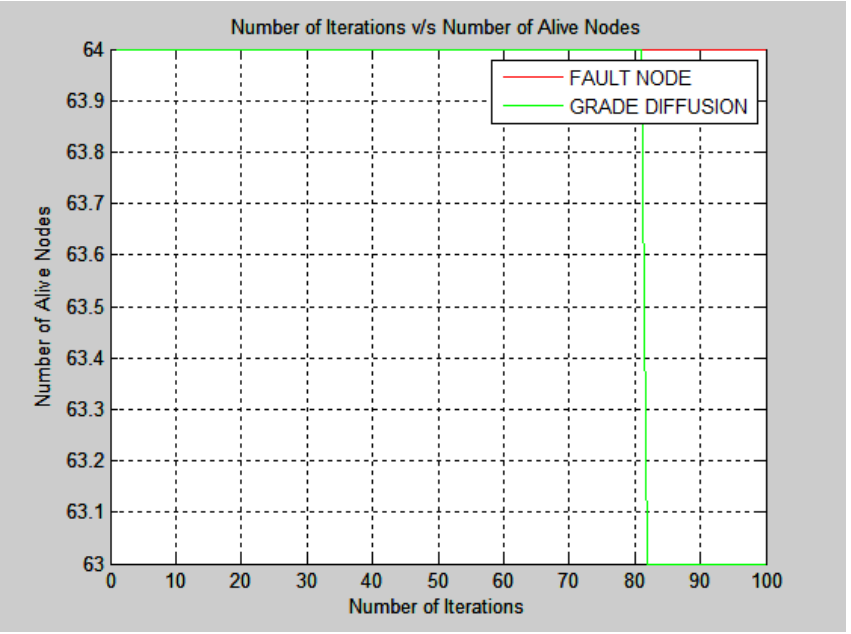

Fig.8. comparsion of number of Alive nodes in fault node recovery algorithm with grade diffusion algorithm

Fig.8 shows the Number of Alive Nodes for the routes discovered using Grade Diffusion and Fault Node and from the results one can prove Fault Node has more Aive Nodes as compared of Grade Diffusion.

\section{Node Life Time}

Node life time is the time at which first dead node occurs in the network.

1. Node Life Time of Fault Node Recovery = Infinity

2. Node Life Time of grade diffusion $=0.008848$

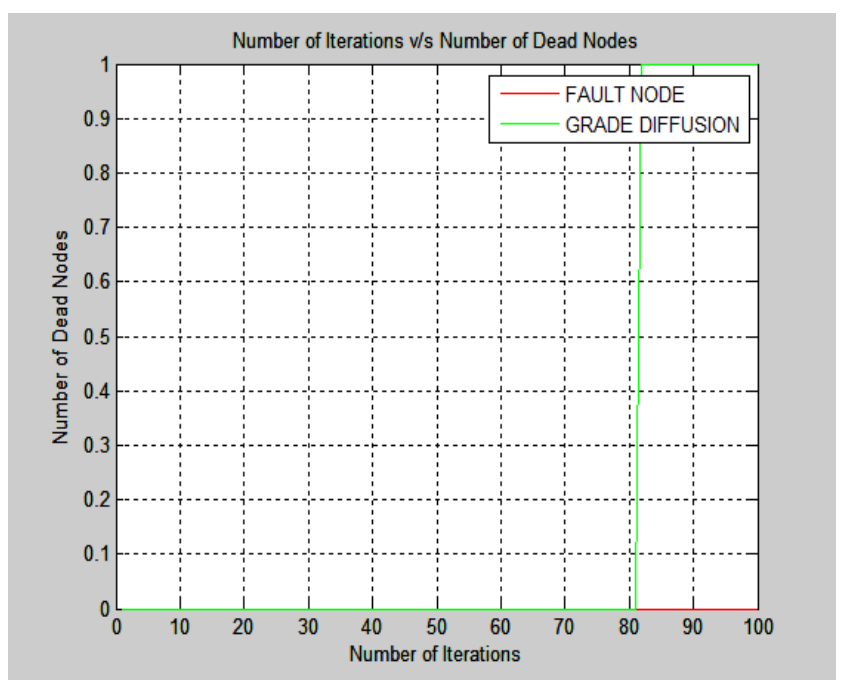

Fig.9.comparsion of number of dead node in fault node recovery algorithm with grade diffusion algorithm

Fig.9 shows the Number of Dead Nodes for the routes discovered using Grade Diffusion and Fault Node and from the results one can prove Fault Node hasless DeadNodes as compared of Grade Diffusion 


\section{CONCLUSIONS}

In real wireless sensor networks, the sensor nodes usebattery power supplies and thus have limited energy resources. In addition to the routing, it is important to research the optimization of sensor node replacement, reducing the replacement cost, and reusing the most routing paths when some sensor nodes are nonfunctional.

This paper proposes a fault node recovery algorithm for WSN. The FNR algorithm requires replacingfewer sensor nodes and reuses the most routing paths, increasing the WSN lifetime and reducing the replacement cost.

The various simulations demonstrate that the time taken, Number of Hops. Power Consumption in $\mathrm{mW}$, Energy Consumption in mJ, Number of Alive Nodes, Number of Dead Nodes, Node lifetime

\section{REFERENCES}

[1]. C. Intanagonwiwat, R. Govindan, D. Estrin, J. Heidemann, and F. Silva, "Directed diffusion for wireless sensor networking," IEEE/ACM Trans. Netw., vol. 11, no. 1, pp. 2-16, Feb. 2003.

[2]. W. H. Liao, Y. Kao, and C. M. Fan, "Data aggregation in wireless sensor networks using ant colony algorithm," J. Netw. Comput. Appl., vol. 31, no. 4, pp. 387-401, 2008

[3]. H. C. Shih, S. C. Chu, J. Roddick, J. H. Ho, B. Y. Liao, and J. S. Pan, "A reduce identical event transmission algorithm for wireless sensor networks," in Proc. 3rd Int. Conf. Intell. Human Comput.Interact., 2011, pp. 147-154.

[4]. T. H. Liu, S. C. Yi, and X. W. Wang, "A fault management protocol for low-energy and efficient wireless sensor networks," J. Inf. Hiding Multimedia Signal Process., vol. 4, no. 1, pp. 34-45, 2013.

[5]. Hong-Chi Shih, Jiun-HueiHo, Bin-YihLiao,andJengShyang Pan, "Fault Node Recovery Algorithm for aWireless Sensor Network," IEEE Sensors Journal, vol. 13, no. 7, pp. 2683-2689, 2013.

[6]. J. H. Ho, H. C. Shih, B. Y. Liao, and S. C. Chu, "A ladder diffusion algorithm using ant colony optimization for wireless sensor networks," Inf. Sci., vol. 192, pp. 204-212, Jun. 2012.

[7]. J. H. Ho, H. C. Shih, B. Y. Liao, and J. S. Pan, "Grade diffusion algorithm," in Proc. 2nd Int. Conf. Eng. Technol. Innov., 2012, pp. 2064-2068.

[8]. S. Corson and J. Macker, Mobile Ad Hoc Networking (MANET): Routing Protocol Performance Issues and Evaluation Considerations. New York, NY, USA: ACM, 1999 [9]. T. P. Hong and C. H. Wu, "An improved weighted clustering algorithm for determination of application nodes in heterogeneous sensor networks," J. Inf. Hiding Multimedia Signal Process., vol. 2,pp. 173-184, 2011. 